\title{
Updates in Lung Cancer 2018-What the Pulmonologist Needs to Know
}

\author{
An Expert Interview with M Patricia Rivera \\ University of North Carolina in Chapel Hill, NC, USA
}

DOI: https://doi.org/10.17925/OHR.2019.15.1.20

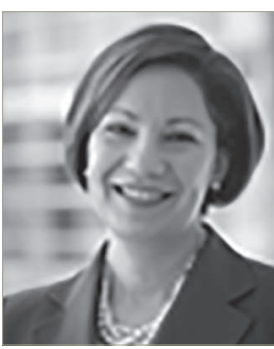

M Patricia Rivera

M Patricia Rivera is Professor of Medicine in the Division of Pulmonary Diseases and Critical Care Medicine at the University of North Carolina at Chapel Hill. She specializes in lung cancer screening, diagnosis, staging, and management of treatment complications. She is Co-director of the Multidisciplinary Thoracic Oncology Program and Director of the Lung Cancer Screening (LCS) Clinic. On the national level Dr Rivera serves as Chair of the Thoracic Oncology Assembly of the American Thoracic Society (ATS) and the Thoracic Oncology Network of the American College of Chest Physicians (ACCP) and is leading research workshops on the impact of co-morbidities on LCS and immunotherapy induced lung disease. She is currently leading a policy statement workshop on disparities in LCS. Dr Rivera is a member of the National Lung Cancer Round Table (NLCRT) serving on the Triage for Appropriate Treatment Task Force, which addresses access to guideline-based care and as Vice Chair of the Lung Cancer in Women Task Force. Throughout her career, Dr Rivera has worked effectively in multidisciplinary clinical and research settings, using her expertise in lung cancer to evaluate evidence-based diagnosis, staging and treatment. Dr Rivera collaborates with colleagues at the University of North Carolina (UNC) through one NCl-funded grant related to lung cancer screening.

\section{Keywords}

Lung cancer, guidelines, immunotherapy, targeted therapy

Disclosure: M Patricia Rivera is Chair of the Scientific and Medical Advisory Committee at bioAffinity Technologies, and is a member of the Scientific and Medical Advisory Committee at Biodesix.

Acknowledgment: Medical writing assistance was provided by Katrina Mountfort of Touch Medical Media, and supported by Touch Medical Media.

Review Process: This is an expert interview and, as such, has not undergone the journal's standard peer review process.

Authorship: The named author meets the Internationa Committee of Medical Journal Editors (ICMJE) criteria for authorship of this manuscript, takes responsibility for the integrity of the work as a whole, and has given final approval for the version to be published.

Received: February 11, 2019

Published Online: May 29, 2019

Citation: Oncology \& Hematology Review. 2019;15(1):20-2

Corresponding Author: M Patricia Rivera, University of North Carolina at Chapel Hill, 130 Mason Farm Road, Suite 4125, Chapel Hill, NC 27599, USA. E: mprivera@med.unc.edu

Support: No funding was received in the publication of this article. ung cancer is the leading cause of cancer deaths in the USA. In 2018 an estimated 154,050 Americans were predicted to die from lung cancer, accounting for approximately $25 \%$ of -all cancer deaths. ${ }^{1}$ However, recent advances have transformed early diagnosis and the treatment landscape for patients with lung cancer. 2018 was an important year; the US Food and Drug Administration (FDA) approved eight new treatment approaches for lung cancer, and updated guidelines have helped clinicians manage patients in all stages of the disease. The eighth edition of the tumor, node and metastasis (TNM) staging system has introduced key changes. Updates to the Fleischner guidelines help facilitate the management of pulmonary nodules. New web-based programs for risk assessment and lung cancer screening implementation are enabling the patient to take an active role in the decision-making process. In terms of treatment, significant progress has been made in the development of drugs targeted to specific gene alterations. However, the most exciting progress in lung cancer continues to be in immunotherapy, which is transforming the treatment landscape for non-small cell lung cancer (NSCLC). In an expert interview, M Patricia Rivera reflects on the key changes in staging, treatment and patient care in 2018.

\section{Q. What are the most important points the pulmonologist needs to know about the new TNM classification for lung cancer and the revised Fleischner Society guidelines for evaluation and management of incidental pulmonary nodules?}

Stage classification, which provides a nomenclature to describe the anatomic extent of the tumor, is fundamental to delivering the appropriate care for patients with lung cancer. The American Joint Committee on Cancer (AJCC) TNM staging system is universally used for the staging of most cancers. The eighth edition of the TNM staging system was implemented in January 2018. ${ }^{2}$ The major changes were in the description of the sizes of the tumors, creating clarity. The primary tumor is now classified into seven categories per size (T1a, T1b, T1c, T2a, T2b, T3, and T4), compared with six previous categories (T1a, $\mathrm{T} 1 \mathrm{~b}, \mathrm{~T} 2 \mathrm{a}, \mathrm{T} 2 \mathrm{~b}, \mathrm{~T} 3$, and $\mathrm{T} 4)$. The other significant change is in classifying metastases: extrathoracic metastatic disease has been split into M1b and M1c components based on single or multiple metastases due to significant differences in survival amongst these categories. One important change was a better way to identify distinct patterns of disease and develop criteria to categorize lung cancer with multiple sites of involvement, for example, synchronous second primary lung cancers, separate tumor nodules (intrapulmonary metastasis), multifocal ground glass/lepidic adenocarcinoma and pneumonic-type adenocarcinoma. These changes have provided a way to better understand the complexity and diversity of the disease. 
The Fleischner guidelines were revised in 2017 and provide an excellent tool for clinicians for the management of pulmonary nodules that are detected incidentally on a computed tomography (CT) scan, based on criteria such as size and appearance of the nodule. Depending on the individual's risk for developing lung cancer, the guidelines provide parameters guiding the clinician in the best way to follow those nodules. Specific topics that have been updated include a new threshold size for follow-up, the importance of the morphologic features of nodules, solid versus subsolid (pure ground glass or part solid lesions), recognition of subsolid components, understanding interval growth or change in nodule morphology, and knowledge of patient risk factors. ${ }^{3}$

\section{Q. What web-based resources are available for lung cancer risk assessment and screening?}

One of the best web resources for lung cancer risk assessment and screening is www.shouldiscreen.com. This program enables the clinician to go through risk factors with the patient in the consulting room. Lung cancer risk is multifactorial; there are many things to consider other than smoking and age, including having chronic obstructive pulmonary disease (COPD), prior history of cancer, and a family history of lung cancer. A risk prediction model calculates an individual's risk of developing lung cancer in the next 6 years and facilitates the discussion of the risks and benefits of screening. In addition, a web-based lung cancer screening implementation guide has been developed in collaboration with the American Lung Association and the American Thoracic Society to help clinicians with the process of implementation of screening programs, shared decision-making, and tobacco counseling.

\section{Q. How is personalized therapy changing the treatment paradigm for lung cancer?}

Personalized therapy in NSCLC has revolutionized patient care. For many years, lung cancer treatment outcomes with systemic chemotherapy had plateaued but that began to change in the early 2000s with the discovery of the role of the epidermal growth factor receptor (EGFR) in lung cancer and more importantly, EGFR mutations as well as subsequent understanding of the molecular heterogeneity of lung cancer. Understanding that certain tumors harbor specific mutations or gene alterations/translocations has led to the development of drugs targeted to specific molecular changes such as EGFR mutations and ALK-4 translocations. Next-generation sequencing (NGS) is now regularly used in practice and liquid biopsies are becoming more widely adopted. ${ }^{4,5}$

Immunotherapy has dramatically changed the treatment landscape of NSCLC. We now have multiple immunotherapy drugs being used in the adjuvant treatment of stage III and in advanced stage NSCLC, and for the first time in decades, we are seeing 5-year survival in patients with metastatic NSCLC. ${ }^{6}$ A key advance in 2018 was the FDA approval of durvalumab for the treatment of patients with locally advanced, unresectable stage III NSCLC who have not progressed following concurrent chemoradiotherapy, the first immunotherapy to be approved for stage III NSCLC. Approval was based on data from the PACIFIC trial, in which consolidation immunotherapy with durvalumab significantly improved progression-free survival and overall survival in patients with stage III NSCLC.? Combined regimens involving chemotherapy, which enhance response of immunotherapies, seem promising, based on data from the KEYNOTE-189 clinical trial of pembrolizumab plus chemotherapy, ${ }^{8}$ and the IMpower150 study of atezolizumab plus chemotherapy. ${ }^{9}$

The changes seen in the 2018 update of the National Comprehensive Cancer Network ${ }^{\circledR}$ (NCCN) Clinical Practice Guidelines in Oncology (NCCN Guidelines) for the treatment of patients with NSCLC reflect the significant progress we have seen in the treatment of lung cancer over the past 12 months. Recent additions include recommendations for osimertinib (category 2A) for the first-line treatment of EGFR-mutant NSCLC, alectinib (category 1) for the first-line treatment of ALK-positive NSCLC, ceritinib (category 2A) for the first-line treatment of ROS-1 positive NSCLC, combination dabrafenib/ trametinib (category 2A) for patients with BRAF V600Emutant NSCLC, pembrolizumab (category 2A) as a single agent for the first-line treatment of patients with NSCLC who have PD-L1 expression of 50\% or greater and in combination with carboplatin and pemetrexed for patients with metastatic NSCLC. ${ }^{10}$

\section{Q. How has the management of complications related to lung cancer care evolved?}

Along with treatment benefits, immune checkpoint inhibitors are associated with adverse events (AES) including an unusual manifestation of inflammatory diseases that can affect every organ. ${ }^{11}$ The incidence of these toxicities is increasing. Ongoing work is trying to establish factors that place patients at risk for these toxicities, how to best detect them early, mechanisms of action to develop treatments, and whether we can rechallenge patients. Immunotherapy is so impactful that we must treat and manage the toxicities. It is important to remember that the same challenges were met when systemic chemotherapy was first used. Working with specialists in other disciplines is important to ensure a good referral system for patients is they experience toxicities.

The American Society for Clinical Oncology (ASCO) and NCCN have published guidelines on the management of immune-related toxicities in patients treated with immune checkpoint inhibitors. These state that all patients receiving checkpoint inhibitors should be monitored for toxicities. If the patient has grade 2 or higher AEs, the checkpoint inhibitor should be halted until the toxicity is reduced to grade $1 \mathrm{AE}$ or less. Patients with grade 3 toxicities might also require high-dose corticosteroids tapered for at least 4 to 6 weeks. If the toxicity is grade 4 , the checkpoint inhibitor should be discontinued permanently. ${ }^{10,12}$

In summary, these are exciting times for pulmonologists and patients alike. We are only beginning to harness the power of the immune system against cancer. Developing treatments to target additional pathways, refining the use as current biomarkers such as PD-L1, and identifying new biomarkers for patient selection are essentially needed to build on recent advances. After a pivotal year of progress, we have high hopes for 2019. $\square$
1. Centers For Disease Control And Prevention. National Center For Health Statistics. CDC WONDER On-Line Database, Compiled from Compressed Mortality File 1999-2016 Series 20 No. 2V, 2017.

2. Kay FU, Kandathil A, Batra K, et al. Revisions to the Tumor, Node, Metastasis staging of lung cancer (8(th) edition): Rationale, radiologic findings and clinical implications. World J Radiol. 2017;9:269-79
3. Bueno J, Landeras L, Chung JH. Updated Fleischner Society Guidelines for Managing Incidental Pulmonary Nodules: Common Questions and Challenging Scenarios. Radiographics. 2018;38:1337-50

4. Garinet S, Laurent-Puig P, Blons $\mathrm{H}$, et al. Current and Future Molecular Testing in NSCLC, What Can We Expect from New Sequencing Technologies? J Clin Med. 2018;7:144.
5. Zhang $\mathrm{Y}$, Zheng $\mathrm{H}$, Zhan $\mathrm{Y}$, et al. Detection and application of circulating tumor cell and circulating tumor DNA in the non-small cell lung cancer. Am J Cancer Res. 2018;8:2377-86.

6. Gettinger S, Horn L, Jackman D, et al. Five-Year Follow-Up of Nivolumab in Previously Treated Advanced Non-Small-Cell Lung Cancer: Results From the CA209-003 Study. I Clin Oncol. 2018;36:1675-84. 


\section{Expert Interview Lung Cancer}

7. Antonia SJ, Villegas A, Daniel D, et al. Durvalumab after Chemoradiotherapy in Stage III Non-Small-Cell Lung Cance. N Eng/ J Med. 2017;377:1919-29.

8. Gandhi L, Rodriguez-Abreu D, Gadgeel S, et al. Pembrolizumab plus Chemotherapy in Metastatic Non-Small-Cell Lung Cancer. N Eng/ J Med. 2018:378:2078-92.
9. Socinski MA, Jotte RM, Cappuzzo F, et al. Atezolizumab for First-Line Treatment of Metastatic Nonsquamous NSCLC N Eng/ J Med. 2018;378:2288-301.

10. NCCN. NCCN Clinical Practice Guidelines in Oncology (NCCN Guidelines $\left.{ }^{\circledR}\right)$ Non-Small Cell Lung CancerVersion 3.2019 -

January 18, 2019. Available at: www.nccn.org/professionals/ physician_gls/recently_updated.aspx (accessed 22 March 2019).
11. Johnson DB, Chandra S, Sosman JA. Immune Checkpoint Inhibitor Toxicity in 2018. JAMA. 2018;320:1702-3.

12. Brahmer JR, Lacchetti C, Schneider BJ, et al. Management of Immune-Related Adverse Events in Patients Treated With Immune Checkpoint Inhibitor Therapy: American Society of Clinical Oncology Clinical Practice Guideline. I Clin Oncol. 2018:36:1714-68. 